COMMENT: NMDAR-Ab encephalitis is a dyskinetic form of encephalitis lethargica that may affect very young children and is rarely preceded by infection. Detectable ovarian tumors are uncommon in female patients under the age of 20 years. Outcome is poor, and early immunotherapy is considered warranted. The detection of specific antibodies is important in children with symptoms of encephalitis. The authors previously reported 20 cases of encephalitis lethargica with evidence of basal ganglia autoimmunity, mainly in children, and the condition is not rare. (Brain 2004;127:21-33; Ped Neur Briefs Jan 2004;18:1-2).

\title{
INCREASED PREVALENCE OF DYSKINETIC CEREBRAL PALSY
}

The prevalence and severity of dyskinetic cerebral palsy (DCP) in European children born 1976-1996 were analyzed in a multicenter study in Goteborg, Sweden; Cork, Ireland; Tubingen, Germany; and Grenoble, France. Of 578 children with DCP, $70 \%$ were born at term. The prevalence per 1000 live births increased from 0.08 in the 1970 s to 0.14 in the 1990s. In 386 children (70\%) with a birth weight $>2500 \mathrm{~g}$ the increase of DCP was significant ( 0.05 to 0.12 ), whereas neonatal mortality in this birth weight group decreased. Compared to children with bilateral spastic cerebral palsy (BSCP), children with DCP had more severe motor and cognitive impairments: 59\% needed a wheelchair, $24 \%$ walked with aids, and $16 \%$ without aids; and $52 \%$ had severe learning disability. Epilepsy developed in $51 \%$, and severe visual and hearing impairment in $19 \%$ and $6 \%$, respectively. Cognitive impairments increased concurrently with severity of motor deficits. In children born 1991-1996, perinatal adverse events (Apgar score <5 at $5 \mathrm{~min}$ and convulsions before $72 \mathrm{~h}$ ) had occurred more frequently in DCP children cf those with BSCP. (Himmelmann K, McManus V, Hagberg G, Uvebrant P, KragelohMann I, Cans C, on behalf of the Surveillance of Cerebral Palsy in Europe. Dyskinetic cerebral palsy in Europe: trends in prevalence and severity. Arch Dis Child Dec 2009;94:921-926). (Respond: Kate Himmelmann, Queen Silvia Children's Hospital, SE41685 Goteborg, Sweden. E-mail: kate.himmelmann@vgregion.se).

COMMENT. Professor PO Pharoah, Department of Public Health, Liverpool, $\mathrm{UK}$, in an editorial, comments as follows: Prevalence is determined by a function of incidence and duration of a disease; an increase in duration or survival results in increased prevalence without change in incidence. The authors consider the increase in prevalence of dyskinetic $\mathrm{CP}$ in term infants is significant because there was no concomitant increase among preterm infants. However, preterm infants contributed more to the decrease in neonatal mortality cf term infants, and therefore to the improved duration due to greater survival. The increasing trend in prevalence of dyskinetic $\mathrm{CP}$ may be related to the higher proportion of small for gestational age infants involved and a prepartum etiological factor. Epidemiological data and caveats are difficult to interpret, (Pharoah PO. Arch Dis Child 2009;94:917-918).

Professor TTS Ingram of Edinburgh University, in his classic studies of "Paediatric Aspects of Cerebral Palsy." (E \& S Livingstone, 1964;pp360-1), compares the prevalence of various types of $\mathrm{CP}$ in children under age 15 years in Edinburgh in 1951. Of total cases including all social classes, dyskinetic CP occurred in 0.17 and diplegia in 0.70 per 1000 . Dyskinesia and diplegia are markedly more prevalent in Social 
Classes I and II than in III-V. Survival of kernicteric babies and dyskinetic cases was higher, due to better infant care in Social Classes I and II than in lower social classes.

\section{TREATABLE GLUT 1 DEFICIENCY CHOREA SYNDROME}

A 7-year-old developmentally delayed girl with chorea and a glucose transporter type 1 (GLUT 1) deficiency syndrome is reported from Hospital Universitari Sant Joan de Deu, Barcelona, Spain, and University of Texas Southwestern Medical Centre, Dallas. At 5 years of age she presented with an abrupt flaccidity and loss of ambulation for several minutes, without loss of consciousness. Chorea and mental retardation were associated with paroxysmal ataxia, convergent strabismus, dysarthria, dystonia, and aggravated intellectual disability induced by fasting or exertion. MRI showed cerebral hypotrophy, especially occipital lobes, and enlarged ventricles. EEG showed mild diffuse slowing, maximal posteriorly. Blond glucose and lactate were normal, but CSF glucose and lactate were diminished. DNA sequencing revealed a sporadic, heterogeneous amino acid insertion in the GLUT 1 transporter that probably impaired blood-brain glucose flux. Treatment with the ketogenic diet was followed by brain growth and improved symptomatic outcome by 7 years of age. Intellectual deterioration was unaffected, remaining below the $5^{\text {th }}$ percentile for age. (Perez-Duenas B, Prior C, Ma Q, et al. Childhood chorea with cerebral hypotrophy. A treatable GLUT 1 energy failure syndrome. Arch Neurol Nov 2009;66(11):1410-1414). (Respond: Juan M Pascual MD $\mathrm{PhD}$, University of Texas Southwestern Medical Center, 5323 Harry Hines Boulevard, Mail Code 8813, Dallas, TX 75390. E-mail: Juan.Pascual@UTSouthwestern.edu).

COMMENT. Most patients with GLUT 1 deficiency syndrome present with epilepsy and deceleration of brain growth, and the above case is considered a novel phenotype. Whereas chorea and ataxia were controlled by treatment with the ketogenic diet, intellectual deterioration was not reversed.

\section{TICS, OCD AND ADHD ASSOCIATED WITH BRAIN TUMOR}

Researchers at Children's Hospital of Michigan, Detroit report a boy aged 12 years with a 12-month history of ADHD, OCD, and stimulant-induced tourette syndrome that resolved following surgical removal of a right temporal lobe and basal ganglia oligodendroglioma. He had also developed complex partial seizures and two episodes of secondary generalized tonic clonic seizures. Oxcarbazepine controlled the seizures, and atomoxetine for ADHD was gradually withdrawn after surgery with no relapse of behavior. Radiation therapy was used for the residual tumor that increased in size 2 years after lesionectomy. Neuropsychological testing 3.5 years after surgery indicated normal verbal intellectual, memory, receptive language, and achievement, with impaired nonverbal, naming, and manual dexterity. Attentional, OCD, and anxiety symptoms resolved postoperatively. (Luat AF, Behen ME, Juhasz C, Sood S, Chugani HT. Secondary tics or tourettism associated with a brain tumor. Pediatr Neurol Dec 2009;41:457-460). (Dr Chugani, Pediatric Neurology/PET Center, Children's Hospital of Michigan, 3901 Beaubien Blvd, Detroit, MI 48201. E-mail: hchugani@ pet.wayne.edu). 